\section{Carcinogens and DNA}

\section{from Stephen Neidle}

THE physical and chemical properties of the potent diolepoxide carcinogens have been much studied in the hope of determining the molecular basis of their carcinogenic and mutagenic effects. They are known to bind directly to DNA, and this reaction has formed the focus of both experimental and theoretical investigations into the activity of diolepoxides such as benzo(a)pyrene 7,8 dihydrodiol 9,10 oxide, the most important active carcinogenic metabolite of benzo $(a)$ pyrene.

Although theoretical approaches to the likely conformation and chemical reactivity of the four possible stereoisomers of benzo(a)pyrene diolepoxide, using quantum-mechanical methodology, have had great influence on thinking in this field, in many cases the theoretical findings conflict with the experimental evidence and it is fair to say that only some of the recent theoretical treatments are successful or other than trivial in their predictions. The deficiencies and limitations of some approaches are highlighted for example, by their inability to explain satisfactorily the considerably greater tumorigenicity and DNA binding ability of the anti compared with the syn isomer (Brookes Cancer Lett. 6,285 ; 1979), even though the theoretically-derived data indicate that the syn isomer is the more stable (Klopman, Grinberg \& Hopfinger J, theor. Biol. 79, $355 ; 1979)$ and until relatively recently was believed to be the biologically significant one.

Application of the $\phi \times 174$ DNA assay system developed by Harvey and coworkers to a series of diolepoxide carcinogens, has led to the revelation of further deficiencies in some quantum mechanical analyses (Hsu et al. Biochem. biophys. Res. Commun. 87, 416; 88, 1; 1979). This assay system is remarkably effective in correlating inhibition of viral infectivity with carcinogenic potency of the various diolepoxides, yet this correlation was not followed either by predictions of reactivities from molecular orbital theory or by the relative structure positions of the diolepoxide groupings on the hydrocarbon skeletons.

Clearly, studies of the chemicals themselves are not enough to explain their different carcinogenic potencies and other factors such as the geometry and kinetics of their binding to the DNA receptor sites will have to be taken into account.

Benzo(a)pyrene diolepoxide carcinogenesis is generally considered to involve direct binding to DNA, with guanine residues as the major sites of attachment at

Stephen Neidle is a Career Development Awardee of the Cancer Research Campaign, working in the Department of Biophysics, King's College, London. least in terms of stoichiometry. About 10\% of the total binding is to adenine however, and although its significance is unclear, some recent evidence correlates binding to adenines and not to guanines with mouse skin tumorigenicity (Di Giovanni et al. Cancer Lett. 7, 39; 1979), although in this report no adducts have actually been characterised as diolepoxide ones. Adeninemodified regions of DNA are known to have rather different conformational properties in terms of greater local denaturation than their guanine counterparts. The anti form of the diol epoxide can be resolved to two stereoisomers. Their interactions with DNA are, not surprisingly, stereospecific (Meehan \& Straub Nature 277, 410; 1979), with essentially only the $(+)$ isomer binding to doublestranded DNA (to guanines) whereas denatured, single-stranded DNA was equally susceptible to both isomers. These differences in binding ability correlate with biological activity in several cell lines - the $(+)$ isomer being more carcinogenic - and Meehan and Straub suggest that DNA secondary structure is an important determinant in carcinogenicity.

One question of interest in looking at the binding of carcinogens to DNA is whether any specific base sequence is involved. Weinstein, Grunberger and coworkers (Biochemistry, in the press) do to a large extent confirm Meehan \& Straub's findings, with the additional important observation from restriction enzyme analysis of modified SV40 and lambda DNA, that no appreciable specificity of binding in respect of base sequence was apparent. However, subtle and possibly important effects of binding specificity may occur. Probably more crucial to carcinogenicity is the geometry of the binding in relation to subsequent excision repair by endonucleases. Binding to different residues and different chemical groups within a residue dramatically affects excisability. Increased excisability implies attachment on the outside rather than to deeply buried sites within the molecule.

There is no lack of molecular models for the structure of benzo $(a)$ pyrene diol epoxide-modified DNA, some of which owe more to imagination than to reality. The recent suggestions of Beland (Chem.Biol. Interactions 22, 329; 1978) are among the more plausible and detailed with the benzo(a)pyrene chromophore being situated in the DNA minor groove and causing some local denaturation of the double helix. This general type of nonintercalative model is strongly supported by several recent physical measurements on the diol epoxide-DNA complex. Fluorescence probe techniques (Prusik et al. Photochem. Photobiol. 29, 223; 1979; Prusik \& Geacintov Biochem. biophys. Res. Commun. 88, 782; 1979) give data consistent with the benzo(a)pyrene chromophore attached (by N2 of guanine) externally to the DNA double helix, and oriented with at most a $35^{\circ}$ angle to the helix axis. In particular, intercalation (at least in the sense of the classical Lerman model) is ruled out by these workers on account of the accessibility of the chromophore to external probings. Additional evidence for a non-intercalative model is also provided by the new technique of optically-detected magnetic resonance (Lefkowitz et al. FEBS Lett. 105, 77; 1979)

Unfortunately such techniques cannot provide detailed information on the molecular geometry of the modified residues of DNA. The situation is made yet more complex by the growing realisation that DNA structure is not always necessarily of the classical forms. There is now powerful evidence for example, that tracts of alternating pyrimidine-purine residues have highly unusual structures (see for example Nature 282, 680; 1979; News and Views, 283, 11; 1980); it would not be surprising if binding of carcinogens did not sometimes exploit such peculiarities. Molecular model-building of nucleic acidcarcinogen interaction urgently requires input of firm data from the fine structure techniques of X-ray crystallography and NMR in order to achieve credibility. Questions of the relevance of the structural peculiarities mentioned above to differences in excisability and repair, and to carcinogenic and mutagenic processes in general must await future investigations. $\square$

\section{The specificity of plant defences}

\section{from R.A. Dixon \& C.J. Lamb}

WHEN plants are attacked by bacterial or fungal pathogens they synthesise and accumulate nonspecific antimicrobial compounds known as phytoalexins. A few years ago it was established that phytoalexins could be induced to accumulate by glucan and glycoprotein 'elicitor' molecules associated with the cell wall of the invading pathogen (see News \& Views 273, 266; 1978) and this has started a search for the molecular basis of the exquisite specificity seen in the classical 'gene-for-gene' relationship between plant cultivars and physiological races of a pathogen, looked at in terms of elicitors and phytoalexin production.

In the simplest terms the gene-for-gene relationship consists of a gene for 'avirulence' in the pathogen being complemented by a specific one for resistance in the plant - the plant and pathogen are in this case incompatible.

R.A. Dixon is in the Department of Biochemistry, Royal Holloway College, University of London and C.J. Lamb is in the Department of Biochemistry, University of Oxford. 